\title{
Secondary Fluorescence Correction for Quantitative X-ray Microanalysis Integrated in a User-Friendly Framework
}

Yu Yuan ${ }^{1}$, Hendrix Demers ${ }^{2}$, Samantha Rudinsky ${ }^{3}$, Nicolas Brodusch ${ }^{1}$, Mathieu Gendron ${ }^{4}$, Eric Yen ${ }^{4}$, Sabrina Clusiau ${ }^{4}$, Nicolas Piché ${ }^{4}$ and Raynald Gauvin ${ }^{1}$

${ }^{1}$ McGill University, Montreal, Quebec, Canada, ${ }^{2}$ Centre d'excellence en électrification des transports et stockage d'énergie, Hydro-Québec, Varennes, Quebec, Canada, ${ }^{3}$ Steam Instruments, Montreal, Quebec, Canada, ${ }^{4}$ Object Research Systems, Montreal, Quebec, Canada

In scanning electron microscopy (SEM) or electron probe microanalysis (EPMA), the Monte Carlo method is widely used for modeling electron and X-ray transport within specimens. For an accurate simulation, the calculation of secondary fluorescence is necessary, especially for specimens with complex structures. Secondary fluorescence is generated through ionization of atoms by higher energy X-rays, including characteristic X-rays and bremsstrahlung X-rays [1]. Because X-rays can travel farther than electrons, secondary fluorescence may be generated at places far away from the primary interaction volume, which could lead to errors in quantitative or even qualitative analysis [2].

A program has been developed for the secondary fluorescence correction of heterogeneous materials based on MC X-ray, a Monte Carlo program that simulates electron trajectories and computes X-ray intensities $[2,3,4]$. The program utilizes a hybrid model, which uses MC X-ray to obtain the three-dimensional primary X-ray distribution and an analytical model to compute the secondary fluorescence. It includes both the characteristic and bremsstrahlung fluorescence and is applicable to arbitrary heterogeneous samples.

Recently, MC X-ray has been integrated into Dragonfly, a software platform for image analysis that is free for non-commercial use [5]. Dragonfly has been widely used for the inspection and analysis of hyperspectral images and other high-dimensional signals [6]. The integration enables greater user flexibility in the simulated sample domains.

We present here that we have added the secondary fluorescence correction in MC X-ray, which is now available in Dragonfly. The secondary fluorescence correction improves the accuracy of the Monte Carlo simulation in predicting X-ray intensities. The integration into Dragonfly decreases the simulation time and makes it available to download for all users.

Two examples of application are shown. The first example is the secondary fluorescence of a light element in bulk samples. Figure 1 shows the variation of the fluorescence ratio (characteristic and bremsstrahlung) for the $\mathrm{C} \mathrm{K} \alpha$ line with respect to $\mathrm{C}$ weight fraction in a $\mathrm{C}-\mathrm{N}$ alloy. The fluorescence ratio is the ratio of the emitted fluorescence X-ray intensities and the primary X-ray intensities. It is observed that both the characteristic and bremsstrahlung fluorescence ratio decrease with increasing $\mathrm{C}$ weight fraction, and as the beam energy increases, the fluorescence ratio increases.

Figure 2 a) shows the sample structure on the X-Y plane of four Co spherical inclusions embedded in a $\mathrm{Cu}$ matrix. $\mathrm{A}$ is the electron beam position with coordinates $(0,0,0)$, and the four Co inclusions have coordinates of their centers at $(2,2,2),(2,-2,2),(-2,2,2)$ and $(-2,-2,2)$ respectively (unit in $\mu \mathrm{m})$. Figure $2 \mathrm{~b})$ presents the variation of secondary fluorescence intensities (characteristic and bremsstrahlung) for 
the $\mathrm{Co} \mathrm{K} \alpha 1$ line as the change of the inclusion radius at $15 \mathrm{keV}$. In this case, the $\mathrm{X}$-ray range for the $\mathrm{Cu}$ $\mathrm{K} \alpha$ line is $1 \mu \mathrm{m}$ around, and the primary $\mathrm{X}$-ray intensities for the $\mathrm{Co} \mathrm{K} \alpha$ line are 0 for all the inclusion radiuses as shown in Figure $2 \mathrm{~b}$ ). As the inclusion size increases, the secondary fluorescence intensities increase. When the inclusion radius is $1 \mu \mathrm{m}$, the quantitative $\mathrm{X}$-ray analysis of the sample using the k-ratio method would result in a composition of $0.37 \mathrm{wt} \% \mathrm{Co}$ and $99.63 \mathrm{wt} \% \mathrm{Cu}$. The chemical composition of the sample is mistakenly estimated without considering the secondary fluorescence.

More examples of applications and the use of MC X-ray as a plugin of Dragonfly will be presented.

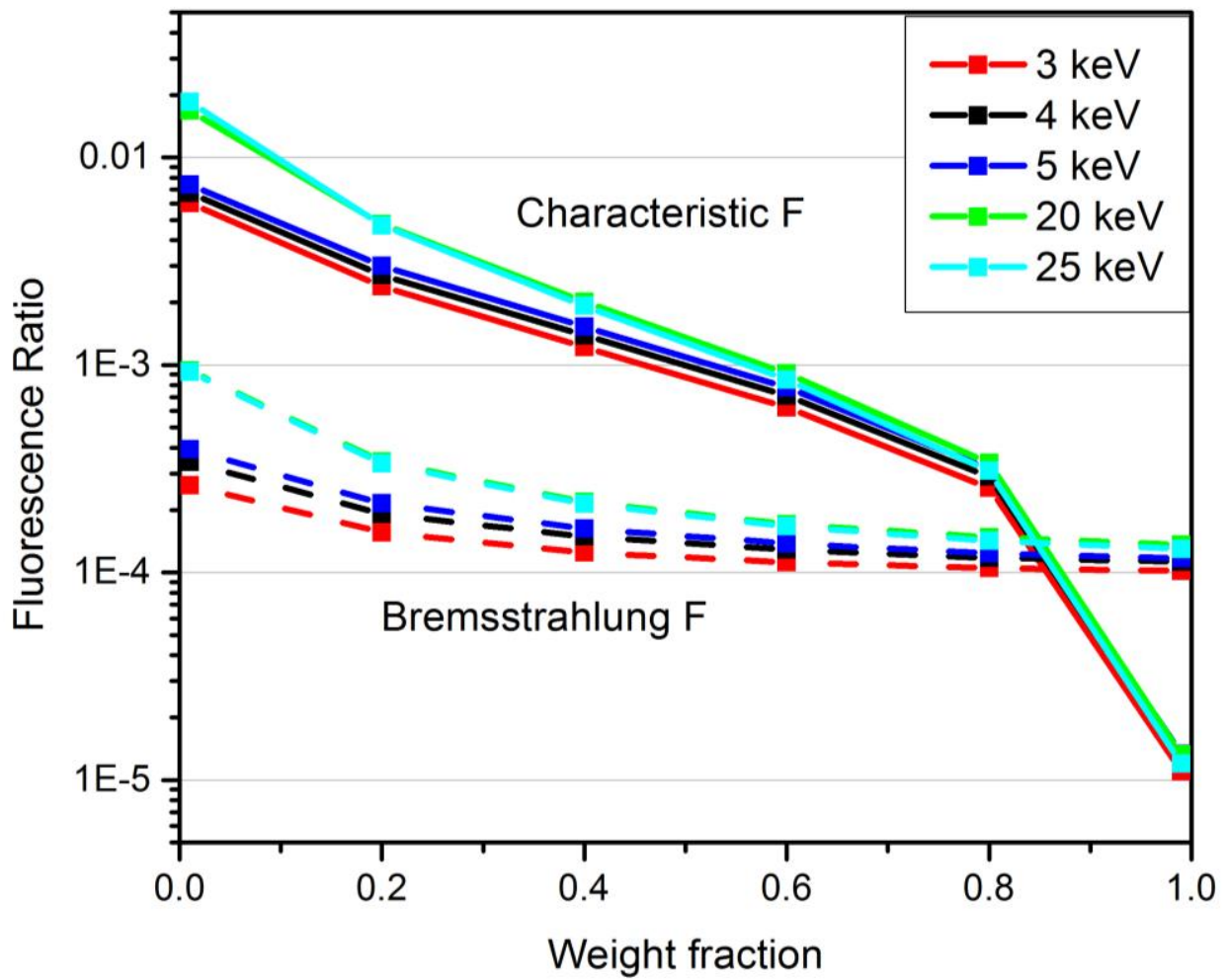

Figure 1. Variation of the fluorescence ratio for the $\mathrm{C} \mathrm{Ka}$ line with respect to the weight fraction of $\mathrm{C}$ in $\mathrm{C}-\mathrm{N}$ alloy at different beam energies.

a)

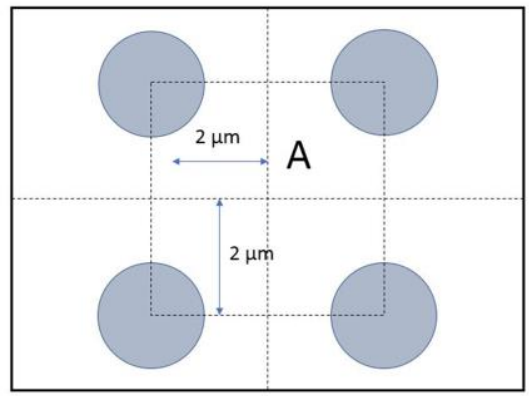

b)

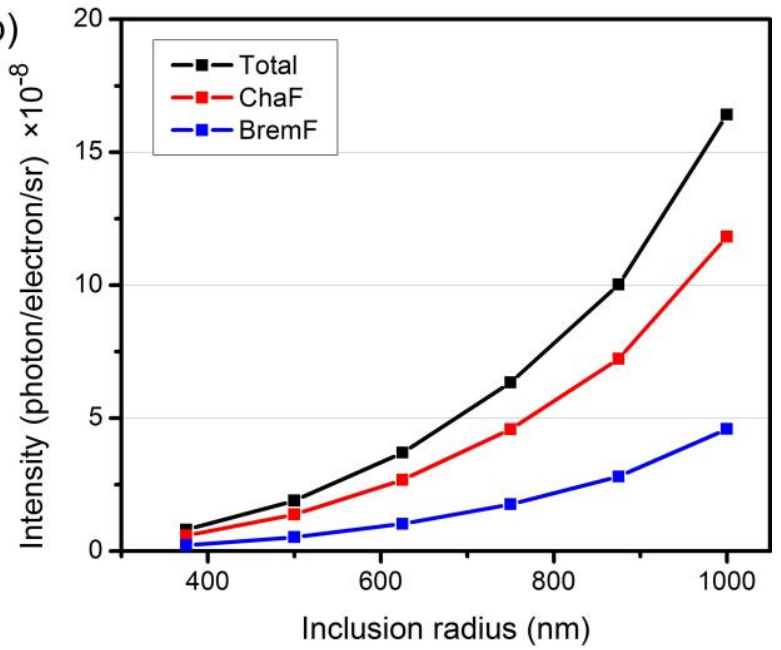


Figure 2. a) Sample structure on the $\mathrm{X}-\mathrm{Y}$ plane for four Co spherical inclusions embedded in a $\mathrm{Cu}$ matrix. b) Variation of the fluorescence $\mathrm{X}$-ray intensities for the Co K $\alpha 1$ line with respect to the inclusion radius at $15 \mathrm{keV}$.

References

[1] J. I. Goldstein, et al., Scanning electron microscopy and X-ray microanalysis, Springer (2017).

[2] Y. Yuan, et al., Microscopy and Microanalysis (2020) (Under review).

[3] Y. Yuan, et al., Microscopy and Microanalysis 25 (1) (2019), p. 92-104.

[4] R. Gauvin and P Michaud, Microscopy and Microanalysis 15 (2009), p. 488-489.

[5] S. Rudinsky, et al., Microscopy and Microanalysis 25 (S2)(2019): p.222-223.

[6] N. Piché, et al., Microscopy and Microanalysis 24 (Suppl 1) (2018), p. 560-561. 